\title{
Cyclovirobuxine D Induces Autophagy-Associated Cell Death via the Akt/mTOR Pathway in MCF-7 Human Breast Cancer Cells
}

\author{
Jing $\mathrm{Lu}^{1}$, Duanping Sun ${ }^{1}, \mathrm{Si} \mathrm{Gao}^{1}$, Ying Gao ${ }^{1}$, Jiantao $\mathrm{Ye}^{1, * a}$, and Peiqing Liu ${ }^{1, * b}$ \\ ${ }^{1}$ Department of Pharmacology and Toxicology, School of Pharmaceutical Sciences, Sun Yat-Sen University, \\ Guangzhou 510006, Guangdong, China
}

Received January 20, 2014; Accepted March 3, 2014

\begin{abstract}
Autophagy is a highly regulated and multi-step biological process that serves to remove damaged cytoplasmic components and organelles. It has been suggested that the activation of autophagy may be a promising therapeutic strategy for cancer treatment by triggering cell death. In this study, we reported that cyclovirobuxine D (CVB-D), an alkaloid component in a traditional Chinese herb, could induce autophagy in the MCF-7 human breast cancer cell line. CVB-D inhibited the viability of MCF-7 cells in a concentration- and time-dependent manner. Activation of autophagy was characterized by transmission electron microscopy, monodansylcadaverine staining, and expression of autophagy marker microtubule-associated protein 1 light chain 3 (LC3). After CVB-D treatment, a clear accumulation of autophagosomes was observed accompanied with elevated LC3 fluorescent puncta. Western blot analysis revealed that CVB-D significantly promoted the conversion from LC3-I to LC3-II and the expression of autophagy-related protein 5 (ATG5), which are both essential for autophagosome formation. On the other hand, CVB-D-induced autophagy and decrease in cell viability could be blocked by 3-methyladenine, a well-established autophagy inhibitor. Moreover, CVB-D attenuated the phosphorylation of Akt and mTOR, two pivotal suppressors in autophagy pathways. These findings shed new light on the pharmacological actions and mechanism of CVB-D and may support the potential utility of autophagy inducers in cancer treatment.
\end{abstract}

Keywords: cyclovirobuxine D, autophagy, breast cancer, Akt, mTOR

\section{Introduction}

Autophagy (meaning self-eating) is a highly regulated, multi-step process by which a cell degrades its own cytoplasmic components and organelles (1). The initial stages of autophagy involve the appearance of intracellular double-membrane autophagic vacuoles, named autophagosomes (2). These autophagosomes then fuse with lysosomes to produce the autolysosomes (3). At this point the sequestered bulk cytoplasm, long-lived proteins, and cellular organelles are degraded by hydrolytic enzymes to re-usable molecules, which are released back into the cell for nutrient recycling and energy

Corresponding authors.

*ayejt@mail.sysu.edu.cn, *bliupq@mail.sysu.edu.cn

Published online in J-STAGE on April 24, 2014

doi: 10.1254/jphs.14013FP supplement (4). Under normal conditions, autophagy remains at a low basal level and plays an essential role in the maintenance of cellular homeostasis, but dysregulated autophagy has been associated with metabolic diseases, inflammatory diseases, neurodegeneration, as well as aging (5).

Increasing evidence has also shown that autophagy is involved in oncogenesis. Some data indicate that autophagy is a survival tool for cancer when the degradation of single cells provides energy for the future development of cancer (6). However, when organelles and portions of the cytoplasm are degraded beyond a certain threshold, autophagic cell death is induced $(7-8)$. Thus, the excessive and sustained activation of autophagy may limit the survival of cancer cells by digesting essential cytoplasmic components (8). It has been reported that the overexpression of genes encoding core components of autophagic pathways, such as Beclin 1, 
can inhibit various cancer types (9), and the induction of autophagy with the use of chemotherapy and radiotherapy helps to remove damaged and mutated cells, thereby reducing tumor size (10). In addition, autophagy is suggested to be an effective approach to alleviate treatment resistance in apoptosis-defective tumor cells (11). Although the definite mechanism underlying autophagic cell death still remains to be elucidated, autophagy can function as a suppressor of cancer, which makes it a promising therapeutic target.

To date, natural products are still present as direct or indirect sources of potential anti-cancer candidates and new chemotherapy adjuvants to enhance the efficacy of chemotherapy and/or to ameliorate its side effects. In China, the medical use of alkaloid-containing traditional herbs has a long history. Growing evidence demonstrates that alkaloids from plants possess anti-cancer activities both in vitro and in vivo by inhibiting proliferation, invasion, and metastasis $(12-14)$. Cyclovirobuxine D (CVB-D), an alkaloid component isolated from the roots of Buxus microphylla var. sinica, is a new drug developed in recent years for the treatment of cardiovascular diseases $(15-16)$. Clinical application shows that CVB-D has beneficial effects on arrhythmia, angina, coronary heart disease, heart failure, and other cardiovascular disorders $(16-19)$. Currently, pharmacological studies on CVB-D have been conducted extensively. However, the role of CVB-D in prevention and treatment of cancer has not been evaluated. In this study, we reported for the first time that CVB-D induced autophagy-associated cell death in MCF-7 human breast cancer cells, which might expand our understanding of the biological activities of CVB-D and support the rational development and exploitation of autophagy inducers in cancer therapy.

\section{Materials and Methods}

\section{Reagents}

CVB-D (purity $\geq 99 \%$, Aladdin Reagent, Shanghai, China) was dissolved in methanol at $10 \mathrm{mM}$ and stored at $4^{\circ} \mathrm{C}$ as a stock solution. Its chemical structure is shown in Fig. 1A. The fluorescent dye monodansylcadaverine (MDC) and 3-methyladenine (3-MA) were purchased from Sigma-Aldrich (St. Louis, MO, USA). Primary antibodies against microtubule-associated protein 1 light chain 3 (LC3) and autophagy-related protein 5 (ATG5) were also from Sigma-Aldrich. Primary antibodies against mTOR, phospho-mTOR (Ser2448), Akt, phospho-Akt (Thr308), and phospho-Akt (Ser473) were products of Cell Signaling Technology (Danvers, MA, USA). Anti- $\alpha$-tubulin primary antibody and all secondary antibodies were purchased from Santa Cruz Biotechnology (Santa Cruz, CA, USA). 3-(4,5-Dimethylthiazol-2-yl)-5-(3-carboxymethoxyphenyl)-2-(4sulfophenyl)-2H-tetrazolium (MTS) was a product of Promega (Madison, WI, USA).

\section{Cell culture}

The MCF-7 cell line was purchased from Cell Bank of Shanghai Institute of Biochemistry and Cell Biology (Shanghai, China). MCF-7 cells were maintained in
A

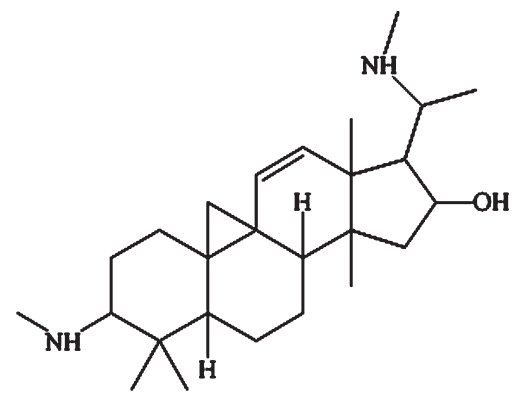

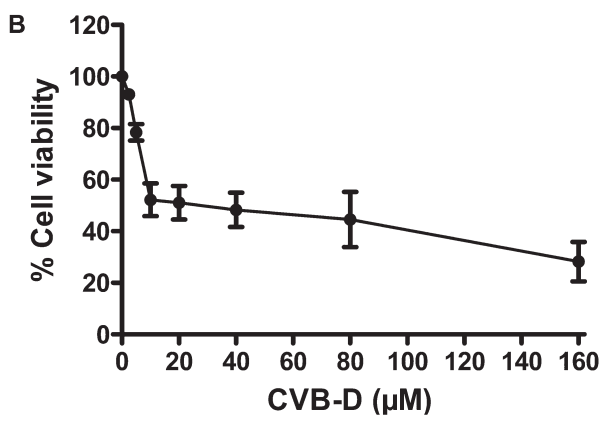

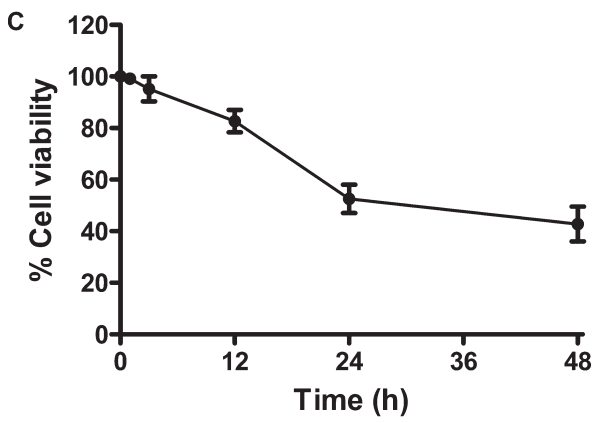

Fig. 1. Effect of CVB-D on cell viability of MCF-7 cells. The chemical structure of CVB-D is shown in panel A. MCF-7 cells were treated with CVB-D $(1-160 \mu \mathrm{M})$ for the indicated time points, and then the cell viability was measured by MTS assay. The viability of the untreated cells was regarded as $100 \%$ (B and C). Data were presented as the mean \pm S.D. of 3 independent experiments. 
Dulbecco's modified eagle medium (DMEM) supplemented with $10 \% \mathrm{v} / \mathrm{v}$ fetal bovine serum (FBS) and $1 \%$ v/v penicillin/streptomycin (GIBCO, Gaithersburg, MD, USA). Cells were grown at $37^{\circ} \mathrm{C}$ in a humidified atmosphere consisting of $5 \% \mathrm{CO}_{2}$. The culture medium was replaced with fresh medium every other day. The cells in the exponential phase of growth were used in the experiments. Cells were incubated with various concentrations of CVB-D for the indicated time points and subsequently submitted to the following tests.

\section{Cell viability}

The cytotoxic effect of CVB-D was determined by MTS assay as described previously (20). MCF-7 cells were seeded at a density of $1.0 \times 10^{4}$ cells/well in 96-well plates. After CVB-D treatment, MTS was added into cell cultures and was incubated for $1 \mathrm{~h}$ at $37^{\circ} \mathrm{C}$. The absorbance was measured at a wavelength of $490 \mathrm{~nm}$ using a microplate reader (Elx800; Bio-Tek, Winooski, VT, USA). Percent viability was defined as the relative absorbance of treated vs. untreated control cells.

\section{Western blotting}

MCF-7 cells were lyzed in RIPA lysis buffer (Beyotime, China) and the protein contents in the supernatant were determined by BCA Protein Assay Kit (Thermo, Waltham, MA, USA). Briefly, the protein was separated by SDS-PAGE gel electrophoresis, and then transferred to polyvinylidene difluoride (PVDF) membranes (Millipore, MA, USA). After blocking with 5\% nonfat milk, the membranes were incubated with primary antibodies [diluted 1:1000 for anti-ATG5, 1:2000 for anti-LC3, 1:1000 for anti-mTOR, 1:1000 for anti-phospho-mTOR (Ser2448), 1:1000 for anti-phospho-Akt (Thr308), 1:1000 for anti-phospho-Akt (Ser473), 1:1000 for anti-Akt, 1:5000 for anti- $\alpha$-tubulin], followed by incubation with appropriate horseradish peroxidase (HRP)-labeled second antibodies (diluted 1:5000). Immunoreactive bands were detected with the Super-Signal West Pico Chemiluminescent Substrate (Pierce, Rockford, IL, USA). The intensity of protein bands was analyzed by LabWorks software (Bio-Rad, Hercules, CA, USA), and $\alpha$-tubulin was used as a loading control.

\section{Immunofluorescence assay}

The cytosolic $18 \mathrm{kDa}$ LC3-I form is converted to the autophagosome-associated $16-\mathrm{kDa}$ LC3-II form by conjugation with phosphatidylethanolamine. This conversion is considered a reliable marker of autophagy (21). MCF-7 cells plated on coverslips were fixed in $4 \%$ paraformaldehyde. After 3 washes with phosphatebuffered saline (PBS), the cells were permeabilized with $0.3 \%$ Triton X-100 for $5 \mathrm{~min}$. Then the cells were incubated at room temperature for $1 \mathrm{~h}$ in blocking solution and further incubated for $1 \mathrm{~h}$ with primary antibodies of LC3 (diluted 1:100). After that, cells were incubated for $1 \mathrm{~h}$ with Alexa Fluor-labeled secondary antibodies (diluted 1:500) and washed with PBS. Coverslips were mounted in Prolong Gold anti-fade reagent with 4',6diamidino-2-phenylindole (DAPI; Invitrogen, Carlsbad, CA, USA) for 2 min and subsequently inspected under a confocal microscope (Zeiss 710; Zeiss, Oberkochen, Germany).

\section{Transmission electron microscopy (TEM)}

To morphologically demonstrate the induction of autophagy in CVB-D-treated tumor cells, the ultrastructural analysis was performed as described previously (22). The cells treated with or without CVB-D were harvested by trypsinization, washed twice with PBS, and fixed with ice-cold glutaraldehyde ( $3 \%$ in $0.1 \mathrm{M}$ cacodylate buffer, $\mathrm{pH}$ 7.4) for $30 \mathrm{~min}$. After washing with $\mathrm{PBS}$, the cells were post-fixed in $\mathrm{OsO} 4$ and embedded in epoxy resin. The 50-nm thin sections were stained with uranyl acetate/lead citrate and viewed in an electron microscope (Hitachi 600; Hitachi, Tokyo).

\section{GFP-LC3 plasmid transfection}

MCF-7 cells expressing LC3 fused to green fluorescent protein (GFP-LC3) were established. Transfection was performed on 100-mm plates with $3 \mu \mathrm{g}$ GFP-LC3 plasmid using Lipofectamine 2000 (Invitrogen) according to the manufacturer's instructions. GFP-LC3 transfected MCF-7 cells were cultured in complete medium and incubated with or without CVB-D for $24 \mathrm{~h}$. Then, the cells were fixed in $4 \%$ paraformaldehyde and analyzed by using a confocal microscope (Zeiss 710).

\section{MDC staining}

Labeling of autophagic vacuoles with fluorescent compound MDC was performed as reported by others (23). MCF-7 cells following CVB-D treatment were incubated with fresh medium containing $0.05 \mathrm{mM}$ MDC. After incubation for $1 \mathrm{~h}$ at $37^{\circ} \mathrm{C}$ in the dark, cells were subsequently washed 3 times with PBS and fixed with $4 \%$ paraformaldehyde for $20 \mathrm{~min}$. Intracellular MDC was measured by a confocal microscope (Zeiss 710 ).

\section{Statistical analysis}

Data were presented as the mean \pm S.D. Statistical analyses between 2 groups were performed by the unpaired Student's $t$-test. Differences among groups were tested by one-way analysis of variance (ANOVA) with Tukey's post hoc test. In all cases, differences were considered statistically significant with $P<0.05$. 

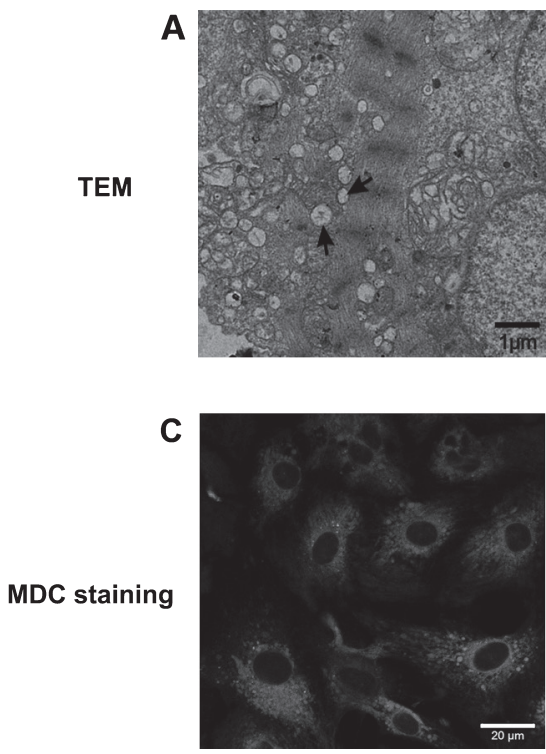

control
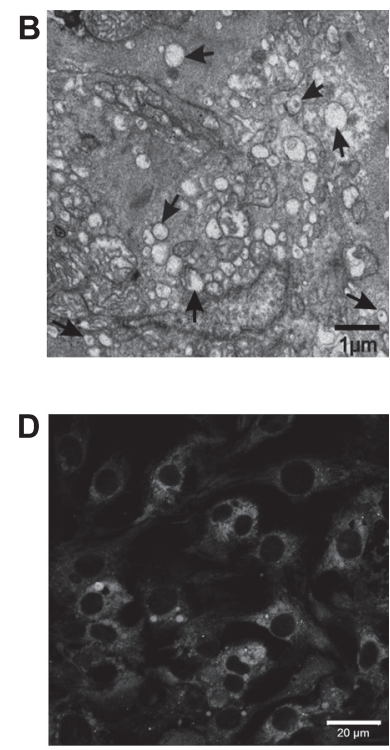

CVB-D
Fig. 2. Induction of autophagy in MCF-7 cells following CVB-D treatment. MCF-7 cells treated with or without CVB-D $(10 \mu \mathrm{M})$ for $24 \mathrm{~h}$ were collected and fixed. The ultrastructure of the cells was detected by TEM (A and B), and the autophagosomes were stained with MDC (C and D), respectively. Representative images out of 3 independent experiments are shown. The arrow indicates an autophagosome including residual digested material in cells. Scale bar: $1 \mu \mathrm{m}$ (A and B); $20 \mu \mathrm{m}$ (C and D).

\section{Results}

Effect of CVB-D on cell viability of $M C F-7$ cell line

To investigate the anti-tumor effect of CVB-D, MCF-7 cells were incubated with 1 to $160 \mu \mathrm{M}$ CVB-D for the indicated time points. Then the cell viability was detected by MTS assay. As shown in Fig. 1, B and C, CVB-D caused a concentration- and time-dependent inhibition of cell growth. The $\mathrm{IC}_{50}$ (concentration resulting in cell viability of $50 \%$ of control) of CVB-D for MCF-7 cells was $10 \pm 2.51 \mu \mathrm{M}$.

\section{Induction of autophagy in MCF-7 cells by CVB-D treatment}

Emerging evidence demonstrates that radiation or some chemotherapeutic agents can promote autophagy in various cancer cells $(25-28)$. Thus, we examined if autophagy was induced in MCF-7 cells in response to CVB-D. After exposure to CVB-D $(10 \mu \mathrm{M})$ for $24 \mathrm{~h}$, MCF-7 cells were collected and the ultrastructure of the cells was detected by TEM. As shown in Fig. 2, $\mathrm{A}$ and $\mathrm{B}$, autophagosomes/autolysosomes were often observed in CVB-D-treated MCF-7 cells, but only a few in untreated cells. Most of the autophagosomes contained lamellar structures or residual digested materials. In addition, MCF-7 cells following CVB-D treatment were incubated with MDC, a compound known to label acidic endosomes, lysosomes, and autophagosomes. Little MDC labeling was present in the control group, whereas marked MDC labeling was observed in the MCF-7 cells treated with CVB-D (Fig. 2: C and D).
These results indicated that MCF-7 cells treated with CVB-D for $24 \mathrm{~h}$ were in the stage of autophagic process.

\section{Involvement of $L C 3$ and ATG5 in CVB-D-treated MCF-7 cells}

LC3, a microtubule-associated protein, is lipidated upon activation of autophagy and associated with the autophagosome membrane (21). To investigate if LC3 was involved in CVB-D-induced autophagy, LC3 puncta formation was assessed by immunofluorescence (IF). The results revealed a clear accumulation of LC3 in the autophagosomes of CVB-D-treated cells (Fig. 3: $\mathrm{A}$ and $\mathrm{B})$. In addition, MCF-7 cells were transfected with GFP-LC3 plasmid. Under a confocal microscope, GFP-LC3-transfected cells showed diffuse distribution of green fluorescence in the absence of CVB-D. In contrast, treatment with CVB-D increased a punctate pattern in number and fluorescence intensity, which represented autophagic vacuoles (Fig. 3: C and D), indicating the recruitment of LC3 in CVB-D-induced autophagy. It is well known that the conversion from LC3-I to LC3-II is a reliable marker of autophagy, and ATG5 is essential for autophagosome formation (21). To further assess autophagy activation in this case, MCF-7 cells following CVB-D treatment were incubated with anti-LC3 and anti-ATG5 antibodies, respectively. The results of western blotting revealed that the protein levels of both LC3-II and ATG5 were significantly upregulated in response to CVB-D treatment (Fig. 3: E and F). 

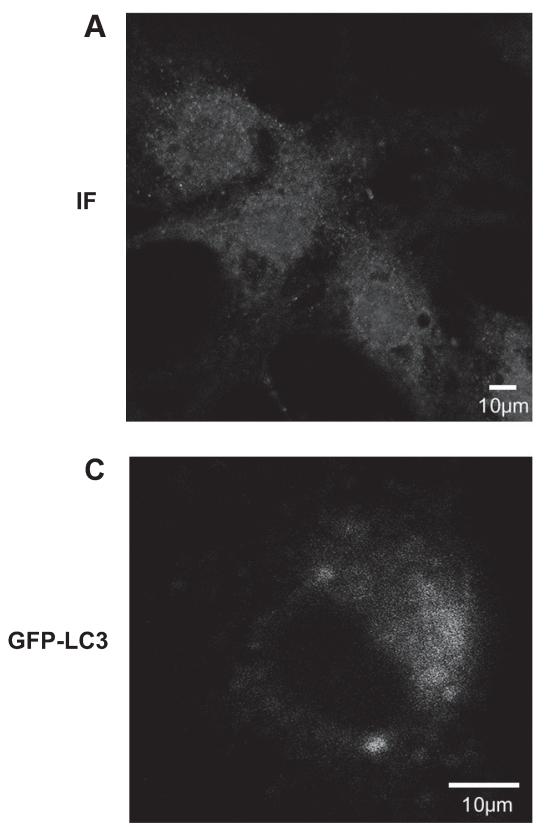

control

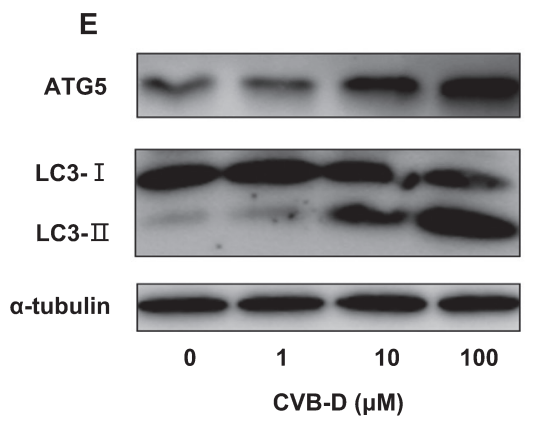

B

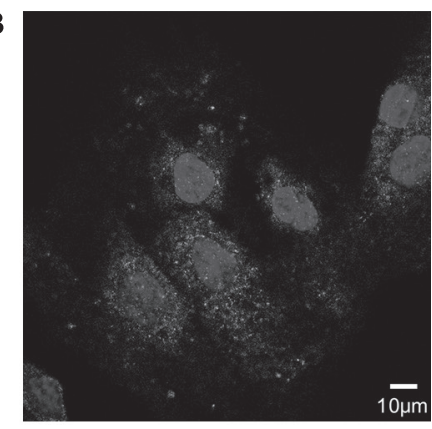

D

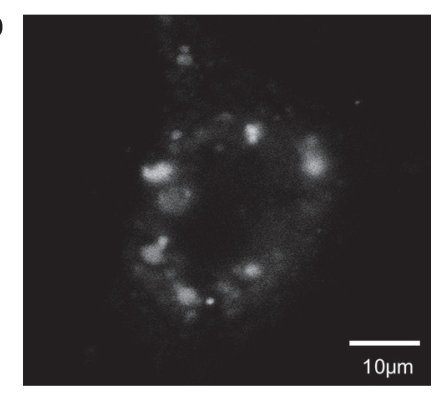

CVB-D

$\mathbf{F}$

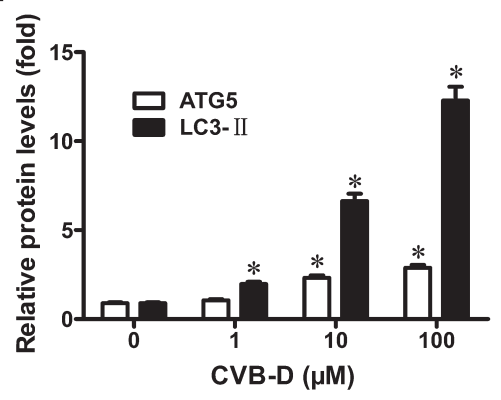

Fig. 3. Involvement of LC3 and ATG5 in CVBD-induced autophagy. A and B) MCF-7 cells were treated with $10 \mu \mathrm{M}$ CVB-D for $24 \mathrm{~h}$. LC3 puncta formation was assessed by immunofluorescence (IF). DAPI was used to stain the nucleus. C and D) MCF-7 cells were transfected with GFP-LC3 plasmid followed by CVB-D treatment. Punctuate signal of GFP-LC3 was observed under a fluorescence microscope. Representative images are shown. Scale bar: $10 \mu \mathrm{m}$. E and F) MCF-7 cells were treated with CVB-D $(1,10,100 \mu \mathrm{M})$ for $24 \mathrm{~h}$. Western blot analysis was performed to detect the expression of autophagy marker ATG5 and the conversion from LC3-I to LC3-II, respectively. The relative protein expression was normalized by $\alpha$-tubulin. Data were presented as the mean \pm S.D. $* P<0.05$ vs. control, $\mathrm{n}=3$.

\section{3-MA blocked CVB-D-induced autophagy and cell death in $M C F-7$ cells}

To investigate the role of autophagy in CVB-Dinduced growth suppression, MCF-7 cells were incubated with the autophagy specific inhibitor 3-MA $(5 \mathrm{mM})$ for $1 \mathrm{~h}$ followed by treatment with $10 \mu \mathrm{M}$ CVB-D. After $24 \mathrm{~h}$, the cells were collected and submitted to western blot analysis. The results showed that the CVB-Dinduced increase in protein expression of LC3-II and ATG5 was markedly reversed by 3-MA (Fig. 4: A and B). Inhibition of autophagy by 3-MA was further examined by MDC staining. As shown in Fig. 4C, 3-MA attenuated the accumulation of MDC-labeled fluorescent particles induced by CVB-D. In addition, MTS assay revealed that 3-MA-pretreatment blocked the inhibitive effect of CVB-D on cell viability as compared to CVB-D treatment alone (Fig. 4D). These observations further indicated that CVB-D promoted MCF-7 cells to autophagic death.

\section{CVB-D inhibited Akt/mTOR phosphorylation in MCF-7 cells}

It has been demonstrated that inhibition of Akt/mTOR axis stimulates autophagy (31). Akt, as a serine-threonine kinase, shows the constitutive activated state in a variety of cancers, which causes phosphorylation of a wide range of downstream targets, and mTOR plays important roles in regulating many anabolic processes including autophagy and cell survival. In this study, MCF-7 cells were treated with CVB-D $(1-100 \mu \mathrm{M})$ for $30 \mathrm{~min}$. Western blot analysis showed that the phosphorylation of Akt (Thr308 and Ser473) was attenuated by CVB-D in a concentration-dependent manner, while the total protein level of Akt remained unchanged as compared to the control (Fig. 5: A and B). Moreover, CVB-D suppressed the phosphorylation of mTOR (Ser2448) without affecting its protein expression (Fig. 5: C and D). These results suggested that the Akt/mTOR axis was inhibited by CVB-D. 
A

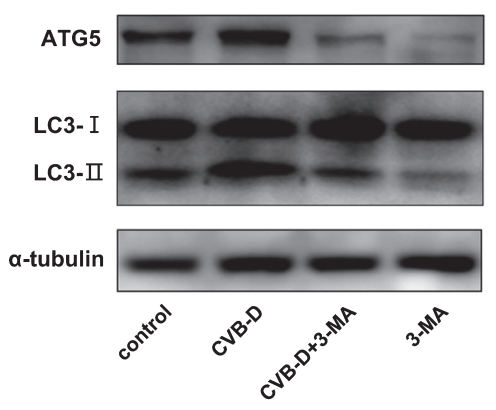

B

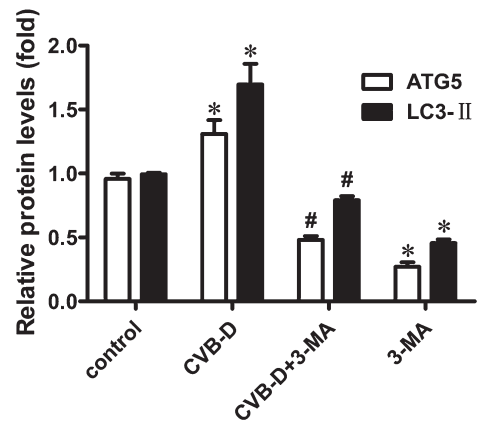

D

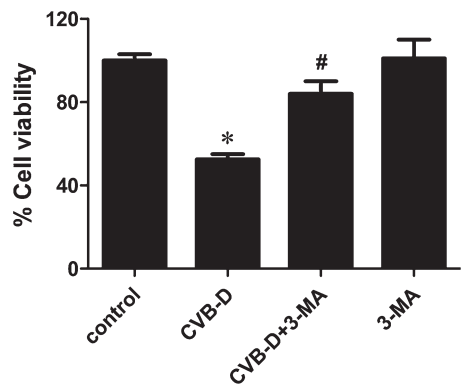

C
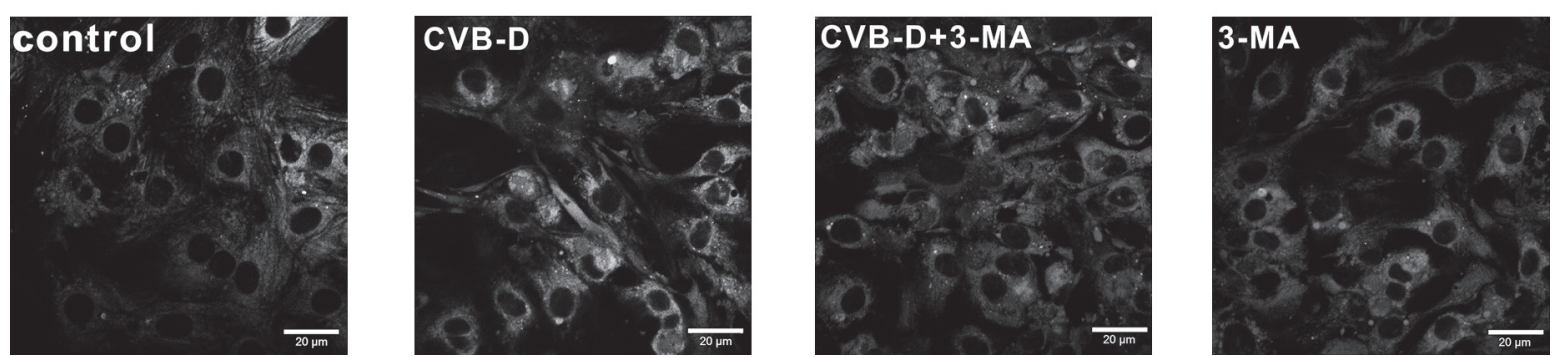

Fig. 4. 3-MA blocked CVB-D-induced autophagy and cell death in MCF-7 cells. Cells were incubated with $10 \mu \mathrm{M}$ CVB-D for $24 \mathrm{~h}$ with or without pretreatment with 3-MA $(5 \mathrm{mM}$, for $1 \mathrm{~h})$. A and B) The protein levels of ATG5 and LC3-II were detected by western blotting and $\alpha$-tubulin was used as an equal loading control. C) The formation of autophagic vacuoles was detected by punctuate MDC labeling, and representative results from 3 independent experiments are shown. Scale bar: $20 \mu \mathrm{m}$. D) The cell viability was measured by MTS assay. Data are presented as the mean \pm S.D. ${ }^{*} P<0.05$ vs. control, ${ }^{\sharp} P<0.05$ vs. CVB-D groups, $\mathrm{n}=3$.

A

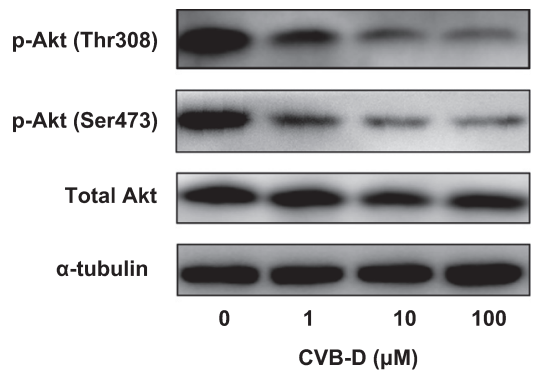

C

p-mTOR (Ser2448)

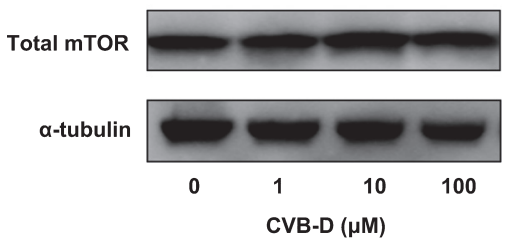

B

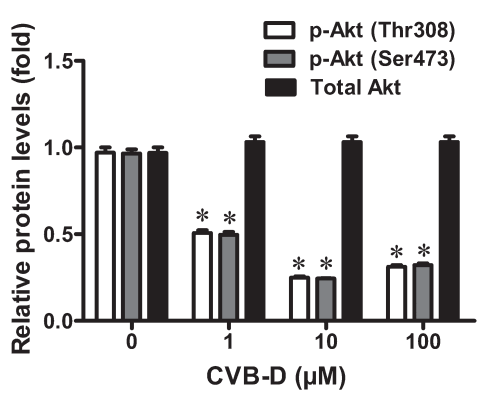

D

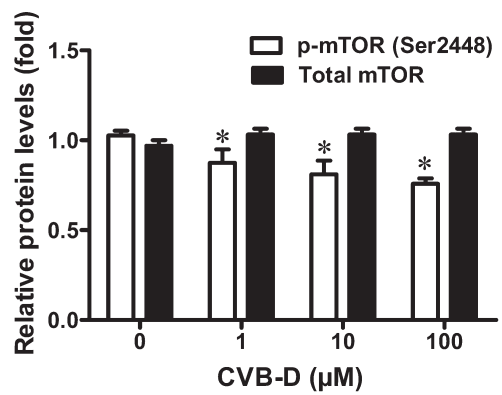

Fig. 5. CVB-D inhibited the phosphorylation of Akt and mTOR. MCF-7 cells were treated with CVB-D $(1,10,100 \mu \mathrm{M})$ for $30 \mathrm{~min}$. The protein levels of Akt, mTOR, phosphoAkt (p-Akt), and phospho-mTOR (p-mTOR) were each determined by western blot. Representative bands are shown (left panels). The relative protein expression was normalized by $\alpha$-tubulin (right panels). Data are presented as the mean \pm S.D. $* P<0.05$ vs. control, $\mathrm{n}=3$.

\section{Discussion}

Currently, autophagy is defined as a biological process in which the cell consumes its own cellular components through the lysosome machinery (24). Upon environmental stress, such as nutrient starvation or pathogen infection, cellular autophagy is activated resulting in either adaption and survival or death. Recently, it 
has been recognized that carcinogenesis is related to decreased levels of basal autophagy. Emerging evidence suggests that the induction of autophagy is a promising therapeutic strategy in cancer treatment. Numerous anti-cancer drugs, such as curcumin, Hirsutanol A, resveratrol, and soybean B-group, have now been identified to exert their cytotoxic actions by inducing autophagic cell death $(25-28)$. Our present study showed that CVB-D, an active compound extracted from a traditional Chinese herb, could promote autophagy in MCF-7 cells, thereby indicating its potential against cancer.

In this study, MTS assay showed that CVB-D induced MCF-7 cell death in a concentration- and time-dependent manner, which was accompanied with parallel increases in the level of autophagy. Here, double membraned and organelle enclosing autophagosomes were identified in CVB-D-treated MCF-7 cells compared to control cells by using TEM. The MDC staining further confirmed a marked activity of autophagy with accumulation of autophagosomes in MCF-7 cells following CVB-D treatment. It's known that $\mathrm{LC} 3$, a mammalian homologue of ATG8 essential for autophagy in yeast, is recruited to the autophagosome membrane in an ATG5-dependent manner (21). Therefore, autophagosome membrane association of LC3 is a specific marker for autophagy. LC3 puncta formation, as assessed by immunofluorescence, revealed a clear accumulation in the CVB-Dtreated cells; and the GFP-tagged LC3-expressing cells were also used to demonstrate induction of autophagy. GFP-LC3 cells presented a diffuse distribution in the control, while a punctate pattern of GFP-LC3 was increased in number and fluorescence intensity by CVB-D. Since LC3 is associated with the formation of autophagosomes through the conversion of LC3-I (cytosolic) to LC3-II (membrane bound), the amount of LC3-II correlates well with the number of autophagosomes and is a well-established indicator of autophagosome formation $(29-30)$. Moreover, the recruitment of LC3 to the membrane occurs via an ATG5-dependent mechanism, and thus ATG5 is essential for autophagosome formation. Western blot analysis revealed that CVB-D significantly up-regulated expressions of both LC3-II and ATG5, further indicting CVBD-induced autophagy in MCF-7 cells.

To explore whether autophagy was involved in the cytotoxicity of CVB-D, the autophagy specific inhibitor 3-MA was used. It has been well established that 3-MA blocks autophagic cell death by inhibiting class III phosphatidylinositol 3-kinase (PI3K) (4). The results of Western blotting and MDC staining showed that autophagy promoted by CVB-D was significantly attenuated by 3-MA. Although 3-MA alone had no toxic effect on MCF-7 cells, it reversed the decrease in cell viability in CVB-D-treated cells. Convergently, these findings suggested CVB-D-induced cell death at least partially through triggering autophagy.

Recent research indicated that the PI3K-Akt-mTOR pathway, which is activated in many types of cancer, is important in autophagy (31). PI3K is a conserved family of lipid kinases that produce lipids involved in cell proliferation, differentiation, apoptosis, autophagy, cytoskeletal organization, and membrane trafficking $(32-34)$. Three classes of PI3K have been defined so far. Class I PI3K is involved in the inhibitory effect on autophagy, while the class III PI3K is involved in the sequestration of cytoplasmic material in autophagy (35). Akt is a serine-threonine kinase, located downstream of class I PI3K, that activates the kinase mTOR, leading to suppression of autophagy (31). As a key negative regulator of autophagy, mTOR lies upstream of all autophagy-associated genes. It, in response to the nutrient levels available to the cell, can inhibit unc-51-like kinase 1 (ULK1) and ATG13 by phosphorylation (36). It is also revealed that mTOR exerts a further brake on autophagy by directly phosphorylating activating molecule in Beclin 1-regulated autophagy (AMBRA1) (37). Rapamycin, which inhibits the mTOR signaling pathway, induces autophagy and inhibits the proliferation of malignant glioma cells (38). Pre-clinical and preliminary clinical studies have demonstrated that targeting mTOR signaling with rapamycin and its analogues is therefore a potent selective therapeutic strategy for many tumor types $(38-39)$. Our data showed that CVB-D did not affect the protein expression of Akt and mTOR, but the phosphorylation of these two proteins significantly decreased following CVB-D treatment. Although mTOR-independent activation of autophagy cannot be excluded, it suggests that inhibition of the Akt-mTOR axis may be involved in autophagic cell death induced by CVB-D.

Taken together, the present work provides evidence that CVB-D promotes autophagic cell death in MCF-7 cells, thereby supporting the potential application of CVB-D in cancer therapy. Certainly, further investigations are required to confirm and clarify the regulation of the PI3K-Akt-mTOR pathway by CVB-D. In vivo studies using cancer animal models are now undergoing in our lab.

\section{Acknowledgments}

This work was supported by grants from the National Natural Science Foundation of China (No. 81072641, No. 81273499, No. 81200096); the National Science and Technology Major Project of China "Key New Drug Creation and Manufacturing Program" (No. 2011ZX09401-307); Team item of Natural Science Foundation of Guangdong Province (No. S2011030003190); and Major Project of 
Department of Education of Guangdong Province (No. CXZD1006), Guangdong Science Foundation (2012A080201007), and Major Project of Guangzhou City (No. 2011Y1-00017).

\section{Conflicts of Interest}

No conflicts of interest are declared by the authors.

\section{References}

1 Kimmelman AC. The dynamic nature of autophagy in cancer. Genes Dev. 2011;25:1999-2010.

2 Yang Z, Klionsky DJ. Mammalian autophagy: core molecular machinery and signaling regulation. Curr Opin Cell Biol. 2010;22:124-131.

3 Levine B, Klionsky DJ. Development by self-digestion: molecular mechanisms and biological functions of autophagy. Dev Cell. 2004;6:463-477.

4 Tsujimoto Y, Shimizu S. Another way to die: autophagic programmed cell death. Cell Death Differ. 2005;12:1528-1534.

5 Shintani T, Klionsky DJ. Autophagy in health and disease: a double-edged sword. Science. 2004;306:990-995.

6 Degenhardt K, Mathew R, Beaudoin B, Bray K, Anderson D, Chen $\mathrm{G}$, et al. Autophagy promotes tumor cell survival and restricts necrosis, inflammation, and tumorigenesis. Cancer Cell. 2006; 10:51-64

7 Liu B, Wen X, Cheng Y. Survival or death: disequilibrating the oncogenic and tumor suppressive autophagy in cancer. Cell Death Dis. 2013;4:e892.

8 Mathew R, Karantza-Wadsworth V, White E. Role of autophagy in cancer. Nat Rev Cancer. 2007;7:961-967.

9 Liang XH, Jackson S, Seaman M, Brown K, Kempkes B, Hibshoosh $\mathrm{H}$, et al. Induction of autophagy and inhibition of tumorigenesis by beclin 1. Nature. 1999;402:672-676.

10 Kanzawa T, Germano IM, Komata T, Ito H, Kondo Y, Kondo S. Role of autophagy in temozolomide-induced cytotoxicity for malignant glioma cells. Cell Death Differ. 2004;11:448-457.

11 Wong VK, Li T, Law BY, Ma ED, Yip NC, Michelangeli F, et al. Saikosaponin-d, a novel SERCA inhibitor, induces autophagic cell death in apoptosis-defective cells. Cell Death Dis. 2013; 4:e720.

12 Jiang X, Huang XC, Ao L, Liu WB, Han F, Cao J, et al. Total alkaloids of Tripterygium hypoglaucum (levl.) Hutch inhibits tumor growth both in vitro and in vivo. J Ethnopharmacol. 2014;151:292-298.

13 Sallam AA, Houssen WE, Gissendanner CR, Orabi KY, Foudah AI, El Sayed KA. Bioguided discovery and pharmacophore modeling of the mycotoxic indole diterpene alkaloids penitrems as breast cancer proliferation, migration, and invasion inhibitors. Med Chem Comm. 2013;4:1360-1369.

14 Kittakoop P, Mahidol C, Ruchirawat S. Alkaloids as important scaffolds in therapeutic drugs for the treatments of cancer, tuberculosis, and smoking cessation. Curr Top Med Chem. 2014;14:239-252.

15 Grossini E, Battaglia A, Brunelleschi S, Mary DA, Molinari C, Viano I, et al. Coronry effects of cyclovirobuxine D in anesthetized pigs and in isolated porcine coronry arteries. Life Sci. 1999;65:59-65.

16 Grossini E, Avanzi G, Gallicchio M, Molinari C, Vacca G,
Bellomo G. Regulation of $\mathrm{Ca}^{2+}$ movements by cyclovirobuxine D in ECV304 endothelial cells. Pharmacol Res. 2005;52: 154-161.

17 Yu B, Fang TH, Lu GH, Xu HQ, Lu JF. Beneficial effect of Cyclovirobuxine $\mathrm{D}$ on heart failure rats following myocardial infarction. Fitoterapia. 2011;82:868-877.

$18 \mathrm{Yu} \mathrm{B}$, Ruan M, Zhou L, Xu L, Fang T. Influence of cyclovirobuxine $\mathrm{D}$ on intracellular $\mathrm{Ca}^{2+}$ regulation and the expression of the calcium cycling proteins in rat myocytes. Fitoterapia. 2012;83:1653-1665.

19 Hu D, Liu X, Wang Y, Chen S. Cyclovirobuxine D ameliorates acute myocardial ischemia by K(ATP) channel opening, nitric oxide release and anti-thrombosis. Eur J Pharmacol. 2007;569: 103-109.

20 Agostini T, Lazzeri D, Pini A, Marino G, Li Quattrini A, Bani D, et al. Wet and dry techniques for structural fat graft harvesting: histomorphometric and cell viability assessments of lipoaspirated samples. Plast Reconstr Surg. 2012;130:331e-339e.

21 Liu Y, Yang Y, Ye YC, Shi QF, Chai K, Tashiro S, et al. Activation of ERK-p53 and ERK-mediated phosphorylation of $\mathrm{Bcl}-2$ are involved in autophagic cell death induced by the c-Met inhibitor SU11274 in human lung cancer A549 cells. J Pharmacol Sci. 2012;118:423-432.

22 Gherghiceanu M, Hinescu ME, Popescu LM. Myocardial interstitial Cajal-like cells (ICLC) in caveolin-1 KO mice. J Cell Mol Med. 2009;13:202-206.

23 Biederbick A, Kern HF, Elsasser HP. Monodansylcadaverine (MDC) is a specific in vivo marker for autophagic vacuoles. Eur J Cell Biol. 1995;66:3-14.

24 Halamoda Kenzaoui B, Chapuis Bernasconi C, Guney-Ayra S, Juillerat-Jeanneret L. Induction of oxidative stress, lysosome activation and autophagy by nanoparticles in human brainderived endothelial cells. Biochem J. 2012;441:813-821.

25 Xiao K, Jiang JH, Guan CX, Dong CL, Wang GF, Bai L, et al. Curcumin induces autophagy via activating the AMPK signaling pathway in lung adenocarcinoma cells. J Pharmacol Sci. 2013; 123:102-109.

26 Yang F, Chen WD, Deng R, Li DD, Wu KW, Feng GK, et al. Hirsutanol A induces apoptosis and autophagy via reactive oxygen species accumulation in breast cancer MCF-7 cells. J Pharmacol Sci. 2012;119:214-220.

27 Puissant A, Robert G, Fenouille N, Luciano F, Cassuto JP, Raynaud S, et al. Resveratrol promotes autophagic cell death in chronic myelogenous leukemia cells via JNK-mediated p62/ SQSTM1 expression and AMPK activation. Cancer Res. 2010; 70:1042-1052.

28 Ellington AA, Berhow M, Singletary KW. Induction of macroautophagy in human colon cancer cells by soybean B-group triterpenoid saponins. Carcinogenesis. 2005;26:159-167.

29 Mizushima N, Yoshimori T, Levine B. Methods in mammalian autophagy research. Cell. 2010;140:313-326.

30 Klionsky DJ, Abdalla FC, Abeliovich H, Abraham RT, AcevedoArozena A, Adeli K, et al. Guidelines for the use and interpretation of assays for monitoring autophagy. Autophagy. 2012;8: $445-544$

31 Grunt TW, Mariani GL. Novel approaches for molecular targeted therapy of breast cancer: interfering with PI3K/AKT/mTOR signaling. Curr Cancer Drug Targets. 2013;13:188-204.

32 Liu Y, Shi QF, Ye YC, Tashiro S, Onodera S, Ikejima T. Activated $\mathrm{O}-2$ (center dot-) and $\mathrm{H} 2 \mathrm{O} 2$ mediated cell survival in SU11274- 
treated non-small-cell lung cancer A549 cells via c-Met-PI3KAkt and c-Met-Grb2/SOS-Ras-p38 pathways. J Pharmacol Sci. 2012;119:150-159.

33 Ma AD, Abrams CS. Pleckstrin homology domains and phospholipid-induced cytoskeletal reorganization. Thromb Haemost. 1999;82:399-406.

34 Dalby KN, Tekedereli I, Lopez-Berestein G, Ozpolat B. Targeting the prodeath and prosurvival functions of autophagy as novel therapeutic strategies in cancer. Autophagy. 2010;6: 322-329.

35 Thi EP, Reiner NE. Phosphatidylinositol 3-kinases and their roles in phagosome maturation. J Leukoc Biol. 2012;92:553-566.

36 Ikeda T, Ishii K, Saito Y, Miura M, Otagiri A, Kawakami Y, et al. Inhibition of autophagy enhances sunitinib-induced cytotoxicity in rat pheochromocytoma PC12 cells. J Pharmacol Sci. 2013;121:67-73.

37 Wang C, Wang Y, McNutt MA, Zhu WG. Autophagy process is associated with anti-neoplastic function. Acta Biochim Biophys Sin. 2011;43:425-432.

38 Ronellenfitsch MW, Steinbach JP, Wick W. Epidermal growth factor receptor and mammalian target of rapamycin as therapeutic targets in malignant glioma: current clinical status and perspectives. Target Oncol. 2010;5:183-191.

39 Zeng ZH, Sarbassov DD, Samudio IJ, Yee KWL, Munsell MF, Jackson CE, et al. Rapamycin derivatives reduce mTORC2 signaling and inhibit AKT activation in AML. Blood. 2007;109: $3509-3512$ 